\title{
Invasive meningococcal disease
}

\section{Doença meningocócica invasiva}

Vanessa L. Strelow, Jose E. Vidal

\begin{abstract}
Invasive meningococcal disease (IMD) is a major public health and continues to cause substantial mortality and morbidity. Serotype $\mathrm{C}$ is the most frequent in Brazil. The clinical spectrum of IMD is broad (meningitis, meningococcemia or both) and the clinical evolution may be unpredictable. Main features associated with mortality are: age higher than 50 years old, seizures, shock, and meningococcemia without meningitis. Blood cultures should be obtained immediately. Lumbar puncture can be performed without previous computed tomography scan (CT) in most cases. Clinical features can be useful to predic patients where an abnormal CT scan is likely. Cerebrospinal fluid (CSF) culture and Gram stain should always be required. Latex agglutination sensitivity is highly variable. Polymerase chain reaction is specially useful when other methods are negative or delayed. Usually ceftriaxone should not be delayed while awaiting CSF study or CT. Dexamethasone can be used in meningococcal meningitis. Early suspicion of IMD and antibiotic in primary care before hospitalization, rapid transportation to a hospital, and stabilization in an intensive-care unit has substantially reduced the case-fatality rate. Vaccines against serotypes A, C, W-135, and Y are available while vaccines against serotype B are expected.
\end{abstract}

Keywords: Neisseria meningitidis, meningococcal meningitis, meningococcal infections, invasive meningococcal disease, bacterial meningitis.

\section{RESUMO}

A doença meningocócica invasiva (DMI) é um problema de saúde pública e continua causando importante mortalidade e morbidade. 0 sorotipo C é o mais frequente no Brazil. O espectro clínico da DMl é amplo (meningite, meningococcemia ou ambos) e a evolução clínica pode ser imprevisível. As principais características associadas a mortalidade são: idade acima de 50 anos, convulsões, choque, e meningococcemia sem meningite. Culturas de sangue devem ser obtidas imediatamente. Punção lombar pode ser realizada sem tomografia computadorizada (TC) prévia na maioria dos casos. Características clínicas podem ajudar a predizer pacientes com elevada probabilidade de apresentar TC alterada. Cultura e Gram no líquido cefalorraquiano devem ser sempre solicitadas. Aglutinação do látex apresenta sensibilidade muito variável. Reação em cadeia da polimerase é especialmente útil quando os outros métodos são negativos ou demorados. 0 uso de ceftriaxona não deve ser retardado enquanto se esperam os resultados do líquor ou TC. Dexametasona pode ser utilizada na meningite meningococóca. Suspeita precoce de DMI, antibiótico no primeira atendimento, antes da admissão hospitalar, transporte rápido para hospital, e estabilização em unidade de terapia intensiva reduz substancialmente a taxa de letalidade. Vacinas contra os sorotipos A, C, W-135, e Y estão disponíveis, entretanto, vacinas contra o sorotipo B são esperadas.

Palavras-Chave: Neisseria meningitidis, meningite meningocócica, infecções meningocócicas, doença meningocócica invasiva, meningite bacteriana.

Invasive meningococcal disease (IMD) is a major public health issue due to its global distribution, potential of epidemic spread, predominant disease burden in children and adolescents, high case-fatality rates and substantial morbidity $^{1-3}$. This disease presents a broad spectrum of manifestations and it is a neurological and clinical emergency that requires prompt recognition and initiation of therapy. In this article, we present a review on the epidemiology, pathophysiology, diagnosis, treatment and prevention of IMD, with focus on its neurological issues.

\section{Microbiology}

Neisseria meningitidis ( $N$. meningitidis) is an exclusively human gram-negative diplococcus that has a great genetic variety $^{1}$. Its genetic plasticity and phenotypic diversity are hallmarks of the meningococcus evolution, as this bacteria acquired various genes from other species of Neisseria and also from other bacterias, like Haemophilus spp. The microorganism is a frequent colonizer of the human nasopharynx and oropharynx, but can also be found in other places, like the oral mucosa, the rectum and the urogenital tract ${ }^{4}$. Meningococcal virulence is related to the major outer membrane components: capsular polysaccharide, outer membrane proteins (pili, porins, Opa, Opc, meningococcal iron-acquiring proteins), and lipo-oligosaccharide (endotoxin) $)^{4-6}$. There are 13 serogroups of $N$. meningitidis based on different capsular polysaccharide structures, but only six serogroups (A, B, C, W-135, Y and more recently $\mathrm{X}$ ) cause most life-threatening disease $\mathrm{e}^{2,6,7}$. 


\section{Epidemiology}

$N$. meningitidis is the main etiological agent of bacterial meningitis in non-neonate children and in young adults ${ }^{8-10}$. According to the Brazilian data from the SIREVA II network, $54 \%$ of cases of invasive disease caused by $N$. meningitidis included in the study in 2010 were children up to 14 years old ${ }^{11}$. Although IMD occurs all over the world, historically, epidemic disease has taken place in Sub-Saharian Africa, known as the African meningitis belt, every 5-10 years since $1905^{6}$. The annual incidence of disease during these epidemics can reach levels up to 1,200 cases per 100.000 inhabitants. In developed countries, such as the USA and in Europe, the disease is mostly endemic (approximately one case per 100.000 inhabitants). Invasive meningococcal disease is endemic in Brazil, with periodic occurrence of outbreaks in several cities. The incidence rates are stable in the recent years, with approximately two cases per 100.000 inhabitants $^{10}$. Endemic disease is caused by a variety of serotypes, whereas epidemics are usually attributable to a single serotype (for example, serogroup A in Sub-Saharan Africa) ${ }^{2}$. However, serotype is not the only responsible for an epidemic: populational susceptibility, agglomerations and poor hygiene conditions all contribute to this event ${ }^{1,2}$. According to the SIREVA II network, the Brazilian distribution of serotypes in 2010 is as follows: serotype $\mathrm{C}$ was responsible for $75 \%$ of the invasive infections, awhile serotypes B, W-135 and Y were responsible for $18 \%, 6 \%$ and $2 \%$ of the cases, respectively ${ }^{11}$.

\section{Pathophysiology}

Human nasopharynx is the only natural reservoir for $N$. meningitidis, and the transmission occurs from one person to another through respiratory secretions. Acquisition of meningococci after very close contact with respiratory secretions or saliva can be transient, can lead to colonization, or result in invasive disease. Colonization of the nasopharynx by the meningococci characterizes carriage. Invasive disease is an infrequent consequence of nasopharynx colonization. Eight to $25 \%$ of healthy individuals may be colonized by $N$. meninditidis for variable periods of time ${ }^{6}$. In times of endemia, approximately $10 \%$ of the population carries the meningococci, although $90 \%$ of these strains are non-pathogenic $^{1,4}$. During epidemics carriage may increase to $18-35 \%$. Carriage rates increase progressively with age, from less than $1 \%$ in children under 4 years old to $25 \%$ in $15-19$ year olds, and up to $32 \%$ in 25 year olds, probably due to changes in social behavior ${ }^{3}$. Most cases of colonization are a result of exposure to asymptomatic carriers and not to patients with $\mathrm{IMD}^{12}$. Colonization rates are higher in agglomerations. Lesions in the nasopharynx epithelium predispose colonization. These lesions may be related, for instance, to smoking, previous viral infections or low air humidity ${ }^{1,4}$. Invasive meningococcal disease is more likely within the first week following the acquisition of a pathogenic meningococcal strain in the nasopharynx ${ }^{1,2}$ Seventy percent of secondary household cases occur within the first week of the index case ${ }^{13}$. The mechanisms that lead from colonization to invasive disease are still incompletely understood but are thought to be a result of meningococcal virulence factors, environmental conditions and host susceptibility ${ }^{3}$.

The highly hydrophilic nature of the bacterial polysaccharide capsule prevents interaction between the meningococci and the epithelial cell. Therefore the production of the capsule must be interrupted to deflagrate invasion ${ }^{2}$. Once in the blood, capsule synthesis reinitiates and this structure is responsible for the protection of the bacteria from phagocytosis ${ }^{1}$.

After invasion, different forms of disease may be established. Virulence is highly related to the release of endotoxins. Patients with low levels of bacteremia may present spontaneous clearance of infection. When it does not happen, the clinical presentation depends on the bacterial properties and host characteristics. The main determinant of the clinical manifestations of meningococcal disease is the extent of activation of the host innate and acquired immune response, which is affected by bacterial factors, such as the amount of circulating endotoxin and bacterial load, as well as by genetic polymorphisms in constituents of the complement system, the inflammatory response and the coagulation and fibrinolytic cascades that affect susceptibility, severity and outcome of the infected individual ${ }^{3}$. Vascular collapse and shock, highly feared complications of meningococcal disease, are related to the effects of the lipo-oligosaccharide, a powerful toxin that activates various immune cells ${ }^{2,6}$.

Most patients ( $>90-95 \%)$ are completely healthy at the moment of the disease. Various genetic polymorphisms were associated to higher risk of infection or worse prognosis in meningococcal disease, such as TNF, Fc $\gamma$ RIIA, Fc $\gamma$ RIII, PAI-1, ACE-1, IL-1Ra, IL-1 $\beta$ and TLR4 ${ }^{4,8,14}$. In addition, an increased incidence of IMD was observed in individuals with deficiencies in the complement system.

\section{Clinical presentations}

In the first 4-6 hours from disease onset, non-specific manifestations mimic the symptoms of common viral infections $^{15}$. Clinical spectrum of IMD is broad and the clinical evolution can be unpredictable: the initial mild febrile illness may progress to fulminant disease, multi-organ failure and death within hours ${ }^{3}$. Neurological presentations can be classified in four major clinical syndromes: (i) meningitis with or without meningococcemia; (ii) meningococcemia without meningitis; (iii) meningoencephalitis with or without meningococcemia; and (iv) bacteremia without sepsis ${ }^{1,2}$. A simpler classification include: (i) meningitis; (ii) meningococcemia; and (ii) meningitis plus meningococcemia. Variations of these scenarios have also been reported, and the patient may progress from one to another during the course of disease. 
The most common presentation of meningococcal disease is meningitis (30-60\% of invasive meningococcal disease), a reflection of the characteristic meningeal tropism of $N$. meningitidis ${ }^{3}$. The classical signs and symptoms of bacterial meningitis are fever, headache, neck stiffness and altered mental status. However, the classical triad of neck stiffness, fever, and altered consciousness is found for only $27 \%$ of patients. Thus, a high level of suspicion is vital to a timely diagnosis.

Children may present initially with only fever and vomits or no specific manifestations. Neck stiffness may be absent early in the course of the illness. Therefore, the absence of this sign should not exclude the diagnosis of bacterial meningitis. In addition, Brudzinski’s sign and Kernig's sign are observed in fewer than $50 \%$ of children with acute bacterial meningitis. Skin lesions typical of IMD (petechiae, purpura, and ecchymoses) are found upon presentation for $60-90 \%$ of children ${ }^{8}$. The rash is typically characterized by progressively enlarging petechial spots that may coalesce into large ecchymotic lesions. It is essential to examine the patient completely undressed ${ }^{2}$.

Most adults with acute bacterial meningitis (>95\%) present with at least two of the following four symptoms: headache, fever, neck stiffness, and altered mental status $^{9,16}$. Brudzinski's sign and Kernig's sign are uncommon. Approximately $60 \%$ of adults with meningococcal meningitis present skin lesions ${ }^{3}$. Focal neurological abnormalities are described in up to $20 \%$ of patients with meningococcal meningitis ${ }^{9}$ (less common when compared to pneumococcal meningitis and meningitis due to Haemophilus influenza) ${ }^{2}$.

Meningococcemia is the predominant presentation in 20$30 \%$ of cases of IMD. Petechial or purpuric rash is seen in 40$80 \%$ of cases. A maculopapular blanching rash is commonly present early in the disease, even among those who later develop a petechial or purpuric rash and persists in $13 \%$ leading to misdiagnosis of a viral infection ${ }^{3}$. Although an ill-appearing child with fever and generalized petechiae had a relevant probability of an invasive bacterial disease, some children with IMD may present non-specific features mimicking a common viral infection. Well appearing children with fever and a non-blanching rash, merit inpatient observation and management. On the other hand, the appearance of a petechial rash in association with fever and drowsiness is highly suggestive of IMD. Confusion and delirium are late signs.

Interestingly, recent data of children under 16 years has shown that general symptoms of sepsis (leg pain, cold extremities, and abnormal skin colour) are frequently seen in the first 12 hours of patients with IMD (median onset 7-12 hours), particularly in severe meningococcaemia, whereas the classic features (haemorrhagic rash, meningism, and impaired consciousness) are relatively late signs (median onset 13-22 hours $)^{15,17}$. Approximately $12 \%$ of patients with IMD have meningitis and meningococcemia ${ }^{15}$. Severe meningococcal septicaemia can progress to purpura fulminans. It is the cutaneous manifestation of disseminated intravascular coagulation.

Uncommonly, other compartmentalized infections may occur, such as septic arthritis, pericarditis and myocar$\operatorname{ditis}^{1,2,8}$. A rare form of disease is known as chronic meningococcemia, which is characterized by recurrent episodes of low grade fever, rash and arthralgia or arthritis, more frequent in adults ${ }^{1}$.

Case-fatality rates of meningococcal disease cases is around $10 \%$ to $20 \%$ in developed countries and remain relatively unchanged over the last decades ${ }^{3,9,18-20}$. Outcomes are different depending on the clinical presentation. For example, in São Paulo and Brazil, overall case-fatality rate of meningococcal disease is approximately $20 \%$. However, figures for meningococcemia, meningitis, and meningococcemia plus meningitis are $37 \%, 10 \%$, and $21 \%$, respectively ${ }^{21}$. As expected, these outcomes can be heterogeneous in function of the characteristics of each institution. For example, a study carried out in a referral center of São Paulo showed a global case-fatality rate of $5 \%$ in patients with meningococcal meningitis ${ }^{22}$. Most patients with fatal IMD die of systemic complications, especially sepsis and septic shock ${ }^{8}$. In this previously mentioned study ${ }^{22}$, when septic shock was present, the case-fatality rate was $30 \%$. In the multivariate analysis, the factors directly associated with in-hospital mortality were: age over 50 years old, seizures, tachycardia, and hypotension.

Post-infectious inflammatory syndromes may complicate $6-15 \%$ of meningococcal meningitis or meningococcemia. These reactions occur from 4 to 12 days after disease onset and include arthritis (most common), cutaneous vasculitis, iritis, episcleritis, pleuritis, and pericarditis. These inflammatory complications are more commonly seen with severe disease, serogroup C N. meningitides, and in adolescents and adults $^{3}$. Survivors of IMD may sustain permanent disabling sequelae ${ }^{3}$. The overall risk of neurological sequelae is $7-12 \%$, lesser when compared to pneumococcal meningitis ${ }^{1,8,23}$. Hearing loss is the most common impairment and occurs in $4 \%^{3}$. Other disabilities include visual impairment, motor deficits, arthritis, spasticity, seizures, learning difficulties, and behavioural and psychological problems ${ }^{3}$.

\section{Laboratorial diagnosis}

Considering the high mortality of acute bacterial meningitis, starting treatment and completing the diagnostic process should be carried out simultaneously in most cases ${ }^{24}$. This concept is applicable to IMD. Laboratorial tests start with obtaining two sets of blood cultures, hemoglobin, WBC and differential counts, platelet count, and coagulation tests. These two last tests are particularly important in patients suspected of developing disseminated intravascular coagulation as a systemic complication of IMD. C-reactive protein 
and serum procalcitonin should be required if available. When confronted with a possible case of IMD, it is mandatory to perform an analysis of the cerebrospinal fluid. In most cases, lumbar puncture can be performed without previous imaging. Computed tomography (CT) of the brain is indicated to detect brain shift either due to focal space-occuping lesion or severe diffuse brain swelling. The incidence of brain herniation after lumbar puncture in patients with acute bacterial meningitis is less than $1 \%^{25}$. Clinical features can be used to predict patients where an abnormal CT scan is likely. The recommended criteria for adult patients with suspected bacterial meningitis who undergo CT of the brain prior to lumbar puncture include: severe immunocompromised state, new-onset seizure, papilledema, abnormal level of consciousness (Glasgow Coma Scale <10), and focal neurological deficit, not including cranial nerve palsies ${ }^{25,26}$. Other contraindications for immediate lumbar puncture are septic shock, severe coagulopathy, skin infection at lumbar puncture site, and spinal cord compression ${ }^{27}$.

Classic cerebrospinal fluid features to any acute bacterial meningitis are present in most cases of meningococcal meningitis: pleocytosis (usually $>1000$ cells $/ \mathrm{mL}$ and $>80 \%$ of polymorphonuclear, but about $20 \%$ can present less of these values), reduction of glucose levels (lower than $40 \%$ of a simultaneously measured serum glucose), and raised protein levels (above $50 \mathrm{mg} / \mathrm{dL}$ ) 8,25 .

A culture showing growth of $N$. meningitidis is still the gold standard method for the diagnosis of meningococcal meningitis and other acute bacterial meningitis ${ }^{2,8}$. Sensitivity of CSF cultures before the start of treatment is high $(>80-90)^{24}$. The main factors for negative cultures in meningococcal disease are administration of antibiotics prior to collecting the material, lack of resources for microbiologic diagnosis and variability of quality in the microbiology services $^{8,28}$. Cerebrospinal fluid cultures became sterile in $90 \%$ to $100 \%$ of patients within 24 to 36 hours of administration of appropriate antimicrobial therapy ${ }^{2}$. Most recently, it has been suggested that cerebrospinal fluid sterilization may occur more rapidly than previously reported, with complete sterilization of cerebrospinal fluid containing meningococci within 2 hours of the first dose ${ }^{2}$. Sensitivity of blood cultures is between $40-80 \%^{8,24}$. For this reason, blood cultures should always be done on admission. Sometimes it is the only diagnostic test available particularly when lumbar puncture cannot be performed. The yield of blood cultures decreases by $20 \%$ if the patient has been pretreated with antibiotics ${ }^{24}$.

Gram stain, a cheap and widely available method, may rapidly identify the causative agent of bacterial meningitis. The exam may be positive even with negative culture results. Positivity may drop slightly with prior antibiotic use. Sensitivity for the detection of meningococcal meningitis is approximately $75 \%$, but could be largely variable, with reported results between $30 \%$ and $89 \%^{2,8,24}$. The specificity of the cerebrospinal fluid Gram stain is above $95 \%{ }^{16}$. Because cerebrospinal fluid Gram stain and culture do not always identify $N$. meningitidis, other methods are necessaries, though they are not routinely available in most resource limited settings.

A fast and widely used diagnostic test for meningococcal meningitis is latex agglutination. However, reported sensitivity is very variable: $22-93 \%$. In addition, lack of sensitivity to detect bacterial antigen has been reported in the cerebrospinal fluid of patients with culture-negative meningitis ${ }^{29}$ and strong decline in the sensitivity (from $60 \%$ to $9 \%$ ) has been reported after treatment ${ }^{8}$.

Polymerase chain reaction (PCR) is increasingly used for diagnosis of meningococcal meningitis including serogrouping and multilocus sequence typing ${ }^{6}$ PCR is specially useful when Gram stain, culture, and/or latex agglutination are negatives. The test improves sensitivity in developed countries and also in Brazil ${ }^{28}$. Its performance is not altered by previous antibiotic use as the method is capable of detecting small amounts of bacterial DNA. PCR's sensitivity and specificity for the diagnosis of meningococcal meningitis using a sample of CSF are between 89-100\% and specificity between $95-100 \%{ }^{24,28}$. Another advantage of the method is the capacity of simultaneous testing for N. meningitidis, Streptococcus pneumoniae and Haemophilus influenzae, and the fact that the results are faster than those of culture ${ }^{2}$.

Although less used in clinical practice, skin biopsies can help the diagnosis of IMD. The skin tissue may undergo Gram stain, culture and histological study ${ }^{8}$. A study reported a sensitivity of $56 \%$ and specificity of $100 \%$ when culture and Gram stain were simultaneously evaluated in skin biopsies of patients with $\mathrm{IMD}^{30}$.

\section{Treatment}

If a patient has clinical signs and/or symptoms suggestive of IMD they should be given parenteral antibiotics immediately after obtaining blood cultures and other blood tests. Therapy should not be delayed while awaiting results of diagnostic tests, such as lumbar puncture or computed tomography scan ${ }^{9,25}$. Thus, early antibiotic treatment should be the primary goal. The experience in some areas of the United Kingdom demonstrated that early suspicion of IMD and prehospital antibiotic in primary care, rapid transportation to a local hospital, and stabilization in an intensive-care unit has substantially reduced the case-fatality rate ${ }^{6}$.

Patients with suspected acute bacterial meningitis are usually given ceftriaxone or cefotaxime. Given the description of $N$. meningitides strains with intermediate resistance to penicillin, and the extreme severity of meningococcal disease, this initial approach seem to be reasonable ${ }^{31}$. When $N$. meningitidis is identified and antibiogram shows full susceptibility, antibiotic treatment can be continued with penicillin or ampicillin ${ }^{2,6,8}$. Although the clinical significance of 
intermediate susceptibility is controversial, in these cases, seems to be more prudent maintain ceftriaxone or cefotaxi$\mathrm{me}^{31}$. There are rare reports around the world of penicillin resistance. In Latin America, the SIREVA II network evaluated the antibiotic susceptibility of strains of $N$. meningitidis. In 2010, of the 506 Brazilian strains tested, 87\% were susceptible to penicillin and $13 \%$ had intermediate susceptibility. None sample was classified as resistant. All strain tested were susceptible to chloramphenicol ${ }^{11}$. Thus, chloramphenicol is an alternative for patients that are allergic to bethalactams ${ }^{2}$. Although there is variability in the literature regarding the ideal duration of antibiotic therapy, classically patients have been treated for 7 days $^{2,6}$.

Corticosteroids are routinely indicated in the initial management of acute bacterial meningitis ${ }^{2,8,32}$. In a recent systematic review, corticosteroids were associated with lower rates of hearing loss and neurological sequelae in acute bacterial meningitis. Subgroup analysis for etiological agent showed that corticosteroids reduced mortality in S. pneumoniae meningitis and reduced severe hearing loss in children with $H$. influenza B meningitis ${ }^{33}$. Another recent study compared two prospective cohorts that evaluated cases of meningococcal meningitis in the Netherlands in two different periods of time: between 1998 and 2002, when the use of dexamethasone in bacterial meningitis was not standardized in the country, and between 2006 and 2011, after the recommendation of dexamethasone in this scenario. In this observational study there was no difference between the overall rates of unfavorable outcome (including mortality and neurological sequelae). The rates of death and hearing impairment were slightly smaller in the period between 2006 and 2011, but there was no statistical significance. Dexamethasone was not associated with adverse events and the rate of arthritis was lower in the group treated with dexamethasone ${ }^{18}$. Taken all together, we suggest the routine use of dexamethasone in meningococcal meningitis. To adults with septic shock and indications of inadequate adrenal function are indicated low doses of steroids ${ }^{6}$.

Symptoms control of post-infectious inflammatory complications is achieved with aspirin or non-steroidal anti-inflammatory drugs and resolution is complete, usually within 14 days from onset, without any residual sequelae ${ }^{3}$.

\section{Prevention}

Aiming to prevent meningococcal disease, numerous vaccines were developed. The first ones were stable and immunogenic vaccines against serotypes A and C. After that vaccines against serotypes $\mathrm{W}-135$ and $\mathrm{Y}$ were developed and have also proven to be safe and immunogenic ${ }^{2}$. Vaccines against all four of these serotypes are available, including quadrivalent ones. In places were these vaccines are implemented for some time, a change in the epidemiology of the disease is notable, with a reduction of incidence of the immunopreventable serotypes ${ }^{12}$. Polysacharide and conjugate vaccines are available ${ }^{34}$. Most vaccines against serotype $\mathrm{B}$, however, demonstrated low immunogenic capacity in humans. Up to this moment, there are no highly effective vaccines to prevent serotype B disease ${ }^{2}$, but new protein-based vaccines are been developed.

The Center for Disease Control and Prevention (CDC) currently indicates meningococcal vaccination for adolescents aged 11 through 18 years and for persons aged under 2 months at increased risk for meningococcal disease, which include persons with medical conditions such as anatomical or functional asplenia or complement component deficiency. Special populations such as unvaccinated or incompletely vaccinated first-year college students living in residence halls, military recruits, or microbiologists with occupational exposure and individuals who travel to or reside in countries in which meningococcal disease is hyperendemic or epidemic. Vaccination is also indicated in outbreaks control. The number of doses required varies according to age and vaccine type ${ }^{34}$.

Chemoprophylaxis is another form of prevention. The aim is to eliminate meningococci from carriers, and thus protect other susceptible individuals. It is recommended for close contacts of patients with the aim of preventing secondary cases since the occurrence of meningococcal disease in household contacts is 100-fold higher than in the normal population $^{6}$. Close contacts include household members, child-care center contacts and anyone directly exposed to the patient's oral secretions) in the 7 days before symptom onset. Because the rate of secondary disease is highest immediately after onset of disease in the index patient, antimicrobial chemoprophylaxis should be administered as soon as possible, ideally within 24 hours of the identification of the index patient ${ }^{34}$. Rifampicin, ceftriaxone, azithromycin, and quinolones all have activity against meningococci in the nasopharynx ${ }^{6}$. Chemoprophylaxis may be administered to patients given penicillin, ampicillin or chloramphenicol for treatment since pharyngeal carriage may not be eliminated with these antibiotics and the patient could remain colonized with a virulent strain. This recommendation, however, is controversial since very few patients harbour disease-causing meningococci after treatment ${ }^{1,6}$.

\section{CONCLUSION}

IMD is associated with serious outcomes, such as death and long-term sequelae. In most cases, lumbar puncture can be performed without previous imaging. Timely antibiotic and rapid transportation to an intensive-care unit can reduced the case fatality rate. Vaccination is available for several serogroups, but vaccines for meningococcal serogroup $\mathrm{B}$ are expected. 


\section{ACKNOWLEDGEMENTS}

We would like to thank Ana Paula Lemos (Instituto Adolfo Lutz, São Paulo State, Brazil) and Camile Moraes
(Departamento de Vigilância Epidemiológica Secretaria de Vigilância em Saúde, Ministério da Saúde do Brasil) for the epidemiological data of meningococcal disease in São Paulo and Brazil.

\section{References}

1. van Deuren M, Brandtzaeg P, Van Der Meer JWM. Update on meningococcal disease with emphasis on pathogenesis and clinical management. Clin Microbiol Rev 2000;13:144-166.

2. Tunkel AR, van de Beek D, Scheld WM. Acute meningitis. In: Mandell, Douglas, and Bennett's Principles and Practice of Infectious Diseases. 7th ed. Churchill Livingstone Elsevier 2010;1189-1229.

3. Pace D, Pollard AJ. Meningococcal disease: clinical presentation and sequelae. Vaccine 2012; 30S:B3-B9.

4. Stephens DS. Biology and Pathogenesis of the evolutionarily successful, obligate human bacterium Neisseria meningitidis. Vaccine 2009:27:71-77.

5. Quagliarello V. Dissemination of Neisseria meningitidis. N Engl J Med 2011;364:1573-1575.

6. Stephens DS, Greenwood B, Brandtzaeg P. Epidemic meningitis, meningococcaemia, and Neisseria meningitidis. Lancet 2007;369: 2196-2210.

7. Racloz VN, Silva JDL. The elusive meningococcal meningitis serogroup: a systematic review of serogroup b epidemiology. BMC Infectious Diseases 2010;10:175.

8. Brouwer MC, Tunkel AR, Van De Beek D. Epidemiology, Diagnosis, and antimicrobial treatment of acute bacterial meningitis. Clin Microbiol Rev 2010;23:467-492.

9. Heckenberg SGB, De Gans J, Brouwer MC, et al. Clinical Features, outcome, and meningococcal genotype in 258 adults with meningococcal meningitis: a prospective cohort study. Medicine 2008;87:185-192.

10. Sáfadi MAP, Cintra OAL. Epidemiology of meningococcal disease in Latin America: current situation and opportunities for prevention. Neurol Res 2010;32:263-271.

11. Organización Panamericana de la Salud. Informe Regional de SIREVA II. 2010. Organización Panamericana de la Salud. Informe Regional de SIREVA II, 2010: datos por país y por grupos de edad sobre las características de los aislamiento de Streptococccus pneumoniae, Haemophilus influenzae y Neisseria meningitidis em procesos invasores. Washington D.C.: OPS 2011;315.

12. Martínez Al, Dominguez A, Oviedo M, et al. Changes in the evolution of meningococcal disease, 2001-2008, Catalonia (Spain). Vaccine 2009;27:3496-3498

13. Salzman MB, Rubin LG. Meningococcemia. Infect Dis Clin North Am 1996;10:709-725.

14. Revest M, Michelet C. Recherce de facteurs favorisants la survenue de méningites bacteriénnes communautaires (nouveau-né exclu). Med Mal Infect 2009;39:562-571.

15. Thompsom MJ, Ninis N, Pereira R, et al. Clinical recognition of meningococcal disease in children and adolescents. Lancet 2006; 367:397-403

16. van De Beek D, de Gans J, Spanjaard L, Weisfelt M, Reitsma JB, Vermeulen M. Clinical features and prognostic factors in adults with bacterial meningitis. N Engl J Med 2004:351:1849-1859.

17. Nascimento-Carvalho CM, Moreno-Carvalho OA. Changing the diagnostic framework of meningococcal disease. Lancet 2006;367: 371-372.
18. Heckenberg SGB, Brouwer MC, van der Ende A, van de Beek, D. Adjunctive dexamethasone in adults with meningococcal meningitis. Neurology 2012;79:1563-1569.

19. Thigpen MC, Whitney CG, Messonier NE, et al. Bacterial meningitis in the United States, 1998-2007. N Engl J Med 2011;364:2016-2025.

20. Levy $\mathrm{C}$, Taha MK, Bingen E, Cohen R. Méningites à méningocoques de l'enfant en France : résultats de l'observatoire ACTIV/GPIP. Archives de Pédiatrie 2012;19:49-54.

21. Ministério da Saúde/SVS - Sistema de Informação de Agravos de Notificação - Sinan Net. Available at: http://dtr2004.saude.gov.br/ sinanweb/index.php. [Accessed on March 20, 2013].

22. Strelow V, Kolbe K, Peixoto de Miranda E, Vidal JE. Meningitis meningocócica (MM) en el Instituto de Infectologia Emílio Ribas (IIER), San Pablo-Brasil, 2006-2011: características clínico-laboratoriales, letalidad y factores asociados a mortalidad intrahospitalaria. XVI Congreso Panamericano de Infectologia. Libro de resúmenes. Santiago de Chile, 2013: 78 .

23. Hoogman M, van de Beek D, Weisfelt M, de Gans J, Schmand B. Cognitive outcome in adults after bacterial meningitis. J Neurol Neurosurg Psychiatry 2007;78:1092-1096.

24. Brouwer M, Thwaites GE, Tunkel AR, van de Beek D. Dilemmas in the diagnosis of acute community-acquired bacterial meningitis. Lancet 2012;380:1684-1692

25. Schut ES, de Gans J, van de Beek D. Community-acquired bacterial meningitis in adults. Prac Neurol 2008;8:8-23.

26. Tunkel AR, Hartman BJ, Kaplan SL, et al. Practice guidelines for the management of bacterial meningitis. Clin Infect Dis 2004;30: $1267-1284$.

27. Roos KL, van de Beek D. Bacterial meningitis. Handbk Clin Neurol 2010;96:52-63.

28. Sacchi CT, Fukasawa LO, Gonçalves MG, et al. Incorporation of realtime PCR into routine public health surveillance of culture negative bacterial meningitis in São Paulo, Brazil. PLoS ONE 2011;6:20675.

29. Tarafdar K, Rao S, Recco RA, Zaman MM. Lack of sensitivity of the latex agglutination test to detect bacterial antigen in the cerebrospinal fluid of patients with culture-negative meningitis. Clin Infect Dis 2001; 33:406-408

30. Arend SM, Lavrijsen AP, Kuijken I, van der Plas RN, Kuijper EJ. Prospective controlled study of the diagnostic value of skin biopsy in patients with presumed meningococcal disease. Eur J Clin Microbiol Infect Dis 2006;25:643-649.

31. VasquezJA.Resistancetesting of meningococci:the recommendations of the European Monitoring Group on Meningococci. FEMS Microbiol Rev 2007;31:97-100.

32. De Gans J, van de Beek D. Dexamethasone in adult with bacterial meningitis. N Engl J Med 2002;347:1549-1556.

33. Brouwer MC, McIntyre P, Prasad K, van de Beek D. Corticosteroids for acute bacterial meningitis. Cochrane Database Syst Rev 2013, Issue 6. Art. No.:CD004405.

34. CentersCenters for Disease Control and Prevention. Prevention and control of meningococcal disease recommendations of the Advisory Committee on Immunization Practices (ACIP). MMRW 2013;62:1-28. 